\title{
Ciguatera au sein de l'équipage d'un navire étranger dans la ville canadienne de Saint John en 2015
}

\author{
Muecke $\mathrm{C}^{1^{*}}$, Hamper $\mathrm{L}^{2}$, Skinner $\mathrm{AL}^{3}$, Osborne $\mathrm{C}^{2}$. \\ ${ }^{1}$ Bureau du médecin-hygiéniste en chef, ministère de la Santé, Fredericton (N.-B.) \\ ${ }^{2}$ Bureau de la protection de la santé de la région du Sud, Bureau du médecin-hygiéniste en chef, Fredericton (N.-B.) \\ ${ }^{3}$ Programme du public voyageur (région de l'Est), Bureau des services de santé à la frontière, Centre de mesures et \\ d'interventions d'urgence, Agence de la santé publique du Canada, Moncton (N.-B.)
}

* Correspondance : cristin.muecke@gnb.ca

\section{Résumé}

L'équipage d'un navire étranger a nécessité des soins médicaux à Saint John, au Nouveau-Brunswick, en raison de l'apparition soudaine de symptômes gastro-intestinaux et dans certains cas, neurologiques et cardiaques, après avoir consommé un repas collectif de poisson. II a été déterminé que la ciguatera était la cause de la maladie. Le présent rapport décrit l'enquête de santé publique et la prise en charge de cet incident, notamment la collaboration entre les autorités provinciales et fédérales concernées.

\section{Présentation du cas}

Le 11 avril 2015, neuf membres de sexe masculin de l'équipage d'un navire étranger accosté à Saint John, au Nouveau-Brunswick, qui présentaient des symptômes gastro-intestinaux (nausée, vomissement, diarrhée et crampes abdominales) se sont rendus au service d'urgence de l'Hôpital régional de Saint John. Parmi les autres symptômes importants, il y avait des étourdissements, des faiblesses et des paresthésies aux extrémités périphériques. Lors de l'examen, on a décelé chez plusieurs des hommes de l'hypotension artérielle et de la bradycardie. Tous ont été admis pour un suivi et une prise en charge aux soins intensifs, qui comprenait un monitorage cardiaque, l'administration d'atropine pour la bradycardie, l'administration d'antiémétiques et une réhydratation intraveineuse. Dans un délai de quelques heures, cinq autres membres de l'équipage qui présentaient les mêmes symptômes se sont aussi rendus à l'hôpital.

Les membres de l'équipage ont indiqué avoir consommé un repas collectif de soupe de poisson, cuisiné à partir d'un poisson qu'ils avaient pêché pendant leur voyage dans les Caraïbes, et qu'ils avaient commencé à se sentir malades d'une à une heure trente plus tard. Les médecins traitants ont consulté le centre antipoisons régional d'Halifax et ont déterminé que la ciguatera était le diagnostic le plus probable. L'incident a été signalé au service de santé publique.

\section{Organismes concernés}

La situation a nécessité une intervention des services de santé publique fédéral et provincial, ainsi que de la régie régionale de la santé. Les rôles respectifs clairs de chaque organisme ont vraisemblablement permis aux mesures de santé publique d'être rapide rapidement mises en œuvre et aux mandats organisationnels ont d'être respectés.

Le Programme du public voyageur (PPV) de l'Agence de la santé publique du Canada (ASPC) est intervenu lors de cette éclosion de maladie entérique dans le cadre de son mandat visant à faire respecter les normes d'hygiène dans les moyens de transport public (dans ce cas-ci, un moyen de transport étranger en eaux canadiennes) (1). Le Programme du public voyageur est également responsable de gérer le Programme de délivrance de certificats de contrôle sanitaire de navire (conformément au Règlement sanitaire international 
de l'Organisation mondiale de la Santé). Le Programme de la quarantaine de l'Agence n'a pas été activé, puisqu'il a été déterminé que l'équipage n'avait pas contracté de maladie transmissible telle que définie dans la Loi sur la mise en quarantaine fédérale.

Les services du Bureau de la protection de la santé du Bureau du médecin-hygiéniste en chef (ministère de la Santé du Nouveau-Brunswick) ont également été retenus, étant donné qu'un événement à déclaration obligatoire a été signalé par le personnel clinique de l'Hôpital régional de Saint John, comme le requièrent la Loi sur la santé publique (2) du Nouveau-Brunswick et ses règlements connexes (3).

La Direction de la préparation aux situations d'urgence et intervention (ministère de la Santé du Nouveau-Brunswick) a fourni un soutien de coordination entre les organismes concernés du système de santé provincial. Le soutien en gestion d'incident clinique et d'urgence a été fourni par Ambulance Nouveau-Brunswick et divers membres du personnel de l'Hôpital régional de Saint John, qui fait partie du Réseau de santé Horizon. Le soutien de laboratoire a été fourni par le laboratoire de chimie alimentaire de l'Agence canadienne d'inspection des aliments (ACIA) à Dartmouth, en Nouvelle-Écosse.

\section{Enquête et méthodes}

Les cliniciens du Nouveau-Brunswick sont tenus de rapporter verbalement les grappes de cas de maladie susceptible d'être d'origine alimentaire ou hydrique à l'intérieur d'une heure à leur bureau de santé publique régional. Ils doivent également signaler les maladies inhabituelles (qui ne surviennent habituellement pas au Nouveau-Brunswick ou qui sont d'une étiologie inconnue) dans un délai de 24 heures. Le personnel en santé publique de garde (l'inspecteur en santé publique et le médecin-hygiéniste en santé publique) a assuré la liaison avec le personnel de l'Hôpital régional de Saint John pour recueillir des renseignements initiaux sur l'état clinique et le diagnostic probable des membres de l'équipage affectés. Les mesures de lutte mises en place comprenaient la fermeture de la cuisine du navire, le suivi des autres membres de l'équipage, l'obtention de mises à jour cliniques régulières sur les membres de l'équipage hospitalisés et la liaison avec les homologues fédéraux concernant l'inspection de la cuisine et du reste des aliments.

Des agents d'hygiène du milieu du Programme du public voyageur ont été dépêchés le 12 avril 2015 pour mener une inspection de la zone de préparation et d'entreposage des aliments sur le navire, prodiguer des conseils sur sécurité des aliments, recueillir des échantillons d'aliments et éliminer tous les produits alimentaires potentiellement contaminés. Des échantillons des restes de poisson du repas suspect et des échantillons des poissons surgelés également pêchés dans les Caraïbes ont été envoyés au laboratoire de l'Agence canadienne d'inspection des aliments (Dartmouth) pour une analyse de l'espèce et des tests sur les toxines.

Des entrevues initiales avec des membres de l'équipage affectés ayant reçu leur congé de l'hôpital ont été effectuées le 14 avril 2015 au moyen d'un questionnaire normalisé sur les maladies entériques. Une lettre décrivant la cause et les mesures de prévention de la ciguatera, ainsi que le pronostic et des conseils sur l'après-maladie, a été remise aux membres de l'équipage le 14 avril 2015. Une entrevue finale des membres de l'équipage affectés a été réalisée conjointement par des responsables fédéraux et provinciaux de l'enquête le 17 avril 2015. Les dossiers médicaux des membres de l'équipage hospitalisés ont été obtenus du Réseau de santé Horizon et examinés pour obtenir des renseignements sur le tableau clinique et l'évolution à l'hôpital.

\section{Résultats}

Dix-neuf membres de l'équipage se trouvaient à bord du navire quand l'éclosion est survenue, et quatorze $(73,7 \%)$ d'entre eux avaient mangé le repas suspect de soupe de poisson. Les cinq autres membres étaient endormis ou ont mangé le repas de porc qui était servi comme substitution. Tous les membres qui ont mangé le repas de poisson ont été malades et ont dû être admis à l'hôpital pour un suivi et une prise en charge aux soins intensifs. Un code orange (incident causant un très grand nombre de victimes corporelles) a été déclenché par l'Hôpital régional de Saint John pour gérer ce flux important de malades en phase critique. 
La cuisine du navire a été fermée jusqu'à ce qu'une inspection complète soit menée, et les autres membres de l'équipage ont obtenu de la nourriture de l'extérieur du navire pendant ce temps. Les autres membres de l'équipage ont été surveillés pendant environ 12 heures, jusqu'à ce qu'on puisse établir avec certitude que personne d'autre n'était susceptible d'être malade.

La durée moyenne du séjour à l'hôpital pour ceux qui ont été affectés a été de 3,5 jours, et une personne a nécessité une réadmission en raison de symptômes continus. À la suite de leur sortie de l'hôpital, les membres de l'équipage ont été rapatriés par les propriétaires du navire, parce que plusieurs d'entre eux ont été jugés « inaptes au travail » par les médecins traitants.

La gravité des symptômes était directement liée à la quantité de soupe de poisson consommée et à la partie du poisson ingérée. Par exemple, la personne qui a présenté les symptômes les plus graves avait mangé la plus grosse portion du repas, y compris la tête du poisson.

Des questions plus en profondeur ont révélé que les membres de l'équipage avaient pêché à la ligne depuis le navire lorsqu'il était ancré aux Bahamas, et qu'ils avaient attrapé plus de 125 kilos de poisson de cette manière. Le poisson a été séparé et surgelé de manière intacte pour la consommation de l'équipage, et des portions ont été mangées quotidiennement lors du voyage vers le nord jusqu'à Saint John, au Nouveau-Brunswick. Au port de Saint John, l'un des plus gros poissons a été décongelé, préparé - les entrailles, les branchies et les nageoires ont été retirées -, écaillé, puis apprêté en une soupe de poisson, la tête comprise.

La soupe de poisson a obtenu un résultat « présumé positif » à la ciguatoxine (en raison d'un manque de normes d'analyse, le laboratoire n'a pas été en mesure de quantifier la concentration de ciguatoxine dans l'échantillon). Le poisson restant sur le navire qui n'était pas étiqueté ou qui avait été obtenu d'un fournisseur alimentaire non approuvé a été plongé dans une solution concentrée d'hypochlorite de sodium pour qu'il soit rendu impropre à la consommation, puis éliminé.

\section{Discussion}

La ciguatera est une maladie d'origine alimentaire causée par l'ingestion de poissons contaminés par une toxine appelée « ciguatoxine ». La ciguatoxine est incolore, inodore et sans goût, et l'apparence du poisson contaminé n'est pas modifiée. Elle ne peut pas être détruite par la cuisson, le fumage, la congélation, la mise en conserve, la salaison ou le séchage. Ce poison est produit par des dinoflagellés, de petits organismes qui s'attachent aux algues poussant dans les zones de récifs océaniques en eaux chaudes. De petits poissons phytophages ingèrent ces algues toxiques, puis sont mangés par de plus gros poissons prédateurs, qui à leur tour sont consommés par des humains (4). Les poissons des zones affectées ne sont pas touchés de manière uniforme; il est donc possible que seulement quelques poissons sur une prise contiennent des niveaux suffisants de toxine pour provoquer une maladie. La toxine est liposoluble et est concentrée dans la tête, les viscères et les œufs du poisson (5). Le système de santé publique a joué un rôle de premier plan dans la transmission de ces renseignements aux membres de l'équipage (et par le fait même, à leurs supérieurs), permettant ainsi de mieux comprendre la cause de la situation et la façon de la prévenir à l'avenir, et fournissant un complément important aux conseils cliniques prodigués à la sortie de l'hôpital.

Les symptômes de la ciguatera peuvent se présenter en quelques minutes, mais ils se manifestent généralement dans les 24 heures suivant la consommation du poisson contaminé. Les symptômes gastro-intestinaux initiaux sont la nausée, le vomissement, la diarrhée et les douleurs abdominales. Ces symptômes peuvent durer plusieurs jours. Les symptômes neurologiques apparaissent après les symptômes gastro-intestinaux initiaux, et comprennent des picotements et un engourdissement autour de la bouche, sur des lèvres, dans la gorge, dans les bras et dans les jambes, des muscles endoloris, une douleur aux dents, une inversion de la température (ce qui est chaud semble froid et vice versa), de la fatigue, des maux de tête et des démangeaisons. Dans les cas graves, les symptômes neurologiques peuvent durer pendant des mois ou plus et peuvent s'aggraver à la suite de changements dans le comportement alimentaire (si la personne suit un régime alimentaire ou si elle consomme des repas à teneur élevée en protéine par exemple), de la consommation d'alcool, de l'exercice ou de relations sexuelles (5). Les renseignements cliniques initiaux et 
le suivi continu des hospitalisations ont constitué un aspect important de l'évaluation de la santé publique; toutefois, des lacunes considérables ont été notées relativement à l'information reçue (comme le manque de rapports sur les symptômes neurologiques et l'absence de procédures systématiques d'analyse des selles), ce qui a entraîné l'examen subséquent des dossiers médicaux. Bien que la participation de plusieurs cliniciens représente un défi, une communication claire des besoins en santé publique, directement entre un médecin en santé publique et un médecin clinique, aiderait à assurer que les préoccupations en santé publique soient prises en compte dans le traitement clinique.

Le diagnostic repose sur une bonne reconnaissance du tableau clinique et des antécédents d'exposition alimentaires compatibles. Aucun test de diagnostic sur des humains n'est disponible actuellement. Cependant, des échantillons de selles devraient être recueillis systématiquement pour éliminer davantage de causes courantes d'intoxications d'origine alimentaire. Si des échantillons de nourriture sont disponibles, ils peuvent être recueillis et analysés pour détecter la présence de la ciguatoxine. Aucun antidote n'existe pour la ciguatera, et les personnes qui ont consommé la ciguatoxine reçoivent un traitement symptomatique (6). La plupart des personnes atteintes de la ciguatera sont guéries en l'espace de quelques jours ou semaines, mais, dans de rares cas, la ciguatera est fatale (4).

L'enquête sur l'éclosion ici exposée représentait une importante occasion d'apprentissage relativement à la dynamique et à la communication entre les organismes. Le principal enjeu fut probablement le chevauchement précoce des rôles des organismes fédéraux et provinciaux dans l'évaluation de la situation et l'intervention initiale. Ce problème a été causé en partie par le fait que les organismes fédéraux et provinciaux ont été avisés par deux processus distincts : dans le cas du Programme du public voyageur, par un avis des services de la quarantaine (qui assure la liaison avec les autorités portuaires), et dans le cas du Bureau de la protection de la santé, par l'hôpital qui a reçu les patients. II en a découlé un chevauchement initial, mais il était clair lorsque nous l'avons revu par la suite, nous avons décidé de ne pas changer le processus car nous en sommes arrivés à la conclusion qu'il est préférable d'avoir un processus redondant au lieu de laisser celui-ci avec des lacunes. Nous avons également conclu qu'il est critique de maintenir des relations continues et des liens entre les organismes afin de s'assurer que les rôles et responsabilités dont clairs pendant une intervention.

Des recherches récentes ont suggéré que les changements climatiques pourraient faire augmenter le nombre de cas de ciguatera en étendant la superficie des habitats propices en eaux chaudes (7), mais il est également possible que si la temperature de la surface de la mer devient trop élevée, la toxigénèse de dinoflagellés chez la ciquatera pourrait diminuer (8). Dans l'intervalle, la prévention repose sur la sensibilisation du public et de l'industrie aux zones et aux espèces touchées par la ciguatera (en particulier, à la nécessité que les aliments destinés aux équipages de navires étrangers soient obtenus uniquement de sources approuvées), puisque les poissons contaminés par la ciguatera ne sont pas facilement détectés et qu'aucune méthode de préparation connue ne peut retirer ou éliminer la toxine.

\section{Remerciements}

Nous souhaitons remercier les personnes suivantes pour leur aide précieuse lors de l'incident :

Denis Belliveau (Programme du public voyageur, Bureau des services de santé à la frontière, Moncton); Tamela Carroll (Bureau du médecin-hygiéniste en chef, Fredericton); Sharf Chowdhury (gestion des urgences, Réseau de santé Horizon); Jessica Crawley (Bureau de la protection de la santé de la région du Sud, Saint John, Nouveau-Brunswick); Carolin Galvin (direction de la Préparation aux situations d'urgence et de l'intervention, ministère de la Santé du Nouveau-Brunswick); Steven Kempton (Mesures et interventions d'urgence, Agence de la santé publique du Canada); Bruce Macfarlane (Communications, ministère de la Santé du Nouveau-Brunswick); Jeff van de Riet (Direction des sciences de la salubrité des aliments, Agence canadienne d'inspection des aliments). 


\section{Références}

(1) Public Health Agency of Canada. Office of Border Health Services [Internet]. Ottawa: Public Health Agency of Canada; 2015. (Disponible en français : www.phac-aspc.gc.ca/cepr-cmiu/ophs-bssp/quar-fra.php)

(2) Public Health Act of New Brunswick. S.N.B. 1998, c. P-22.4. [Internet]. http://laws.gnb.ca/en/browsetitle?letter=P.

(3) New Brunswick Regulation 2009-136 under the Public Health Act. (OC 2099-455) [Internet]. (Disponible en français: http://laws.gnb.ca/fr/browsetitle)

(4) Government of Canada. Ciguatera [Internet]. Ottawa: Government of Canada; 2015. Disponible en français: http://voyage.gc.ca/voyager/sante-securite/maladies/ciguatera

(5) Centers for Disease Control and Prevention (CDC). Ciguatera fish poisoning - Texas, 1997. MMWR Morb Mortal Wkly Rep. 1998 Aug;47(33):692-4.

http://www.cdc.gov/mmwr/preview/mmwrhtml/00054548.htm

(6) Schlaich C, Hagelstein JG, Burchard GD, Schmiedel S. Outbreak of ciguatera fish poisoning on a cargo ship in the Port of Hamburg. J Travel Med. 2012;19(4): 238-42.

(7) Gingold DB, Strickland MJ, Hess JJ. Ciguatera fish poisoning and climate change: Analysis of National Poison Center data in the United States, 2001-2011. Environ Health Perspect. 2014;122(6):580-6.

(8) Llewellyn LE. Revisiting the association between sea surface temperature and the epidemiology of fish poisoning in the South Pacific: Reassessing the link between ciguatera and climate change. Toxicon. 2010;56(5):691-7. 